\title{
On groups with consecutive three smallest character degrees
}

\author{
Shitian Liu ${ }^{\mathrm{a}, *}$, Donglin Lei ${ }^{\mathrm{b}}$, Xianhua $\mathrm{Li}^{\mathrm{b}}$ \\ a School of Science, Sichuan University of Science and Engineeing, Zigong Sichuan 643000 China \\ b School of Mathematical Science, Soochow University, Suzhou, Jiangsu 251125 China
}

*Corresponding author, e-mail: s.t.liu@yandex.com

Received 13 Aug 2018

Accepted 4 Sep 2019

\begin{abstract}
Huppert determined the structure of groups whose degrees are consecutive, then Qian improved that Huppert's result and showed the structure of finite groups whose degrees of nonlinear characters are consecutive. In this paper, we considered the case when the first three smallest degrees of nonlinear irreducible characters of an almost simple group $G$ are consecutive. Furthermore, Huppert's conjecture is proved valid for those groups.
\end{abstract}

KEYWORDS: almost simple group, character degree graph, solvable

MSC2010: 20C15 20C33

\section{INTRODUCTION}

All groups considered here are finite. Let $\operatorname{Irr}(G)$ be the set of all complex irreducible characters of a group $G$, and let $\operatorname{cd}(G)$ be the set of character degrees of a group $G$. Suppose that $n$ and $n+1$, $n>1$, are two consecutive integers standing for the two greatest sizes of the conjugacy classes of group $G$. Then $G / Z(G)=\left(C_{p^{a}-1}, E_{p^{a}}\right)$ is a Frobenius group with kernel $E_{p^{a}}$ and complement $C_{p^{a}-1}, n+1=p^{a}$, and the pre-images in $G$ of the kernels of $G / Z(G)$ are abelian. Meanwhile it determined that the groups with conjugacy classes of lengths $1, n$ or $n+1$ are considered ${ }^{1}$. Several authors have determined the structure of finite groups whose conjugacy class sizes are consecutive ${ }^{2,3}$. Dual to the sizes of the conjugacy classes of a group $G$, what is the influence that character degrees of a group $G$ may have on the structure of a finite group? The influence of the set $\operatorname{cd}(G),|\operatorname{cd}(G)| \geqslant 4$, on the structure of finite groups has been investigated by a number of authors ${ }^{6-11}$.

For a group $G$, if $\operatorname{cd}(G)=\left\{s_{0}, s_{1}, \ldots, s_{t}\right\}$ with $1=$ $s_{0} \leqslant s_{1}<\cdots<s_{t}$, then make $d_{i}(G)=s_{i}$ stand for the $i$ th smallest degree of $G$ for all $1 \leqslant i \leqslant t$. When there is no confusion, we may simply write $d_{i}=s_{i}$. If $t=0$, then $G$ is abelian; if $t=1$, then, for $\operatorname{cd}(G)=$ $\{1, m\}$, either $G$ has an abelian normal subgroup of index $m$, or $m=p^{e}$ for a prime $p$ and $G$ is the direct product of a $p$-group and an abelian group ${ }^{4}$. If $t=$ 2, then $|\operatorname{cd}(G)|=3, G$ is solvable ${ }^{5}$, and $G^{\prime \prime \prime}=1$.

Huppert ${ }^{5}$ proved the following result.
Theorem 1 (Ref. 5) Suppose $\operatorname{cd}(G)=\{1,2, \ldots, k-$ $1, k\}$. Then $G$ is solvable if and only if $k \leqslant 4$; if $k>4$, then $k=6$ and $G=H Z(G)$ with $H \cong S L(2,5)$.

Furthermore, Qian ${ }^{12}$ proved the following results.

Theorem 2 (1) If $G$ is a solvable group whose all nonlinear character degrees are consecutive integers, then one of the following are true:

(i) $|\operatorname{cd}(G)| \leqslant 2$;

(ii) $\operatorname{cd}(G)=\left\{1, p^{m}-1, p^{m}\right\}$ or $\left\{1, p^{m}, p^{m}+1\right\}$;

(iii) $\operatorname{cd}(G)=\{1,2,3,4\}$.

(2) An insolvable group $G$ whose nonlinear character degrees are consecutive integers if and only if $G / Z(G) \cong P G L(2, q)$ for some prime power $q \geqslant 4$.

Hypothesis (C): The three smallest degrees of nonlinear irreducible characters of a group $G$ are consecutive.

Many authors have employed the degrees of irreducible characters to determined the simple groups and some solvable groups. Then what will occur if a group $G$ satisfies Hypothesis (C)?

Recall that a group $A$ is almost simple if there is a simple group $S$ such that $S \leqslant A \leqslant \operatorname{Aut}(S)$. In this paper we only consider the influence of Hypothesis (C) on the structure of almost simple finite groups.

Theorem 3 Let $G$ be an almost simple group and let $L$ be a nonabelian simple group. Assume that $G$ and $L$ satisfy Hypothesis $(C)$, that is, $d_{i}(G)=d_{i}(L)$ for all $i \in\{1,2,3\}$. Then $L=L_{2}\left(2^{m}\right)$ with $m \geqslant 2$, and $G$ has 
a normal subgroup $K$ such that $G / K$ is isomorphic to L. If $|G|=|L|$, then $G \cong L$.

Remark 1 Unfortunately, we cannot determine the structure of solvable groups since there are many examples proven to satisfy the condition of Hypothesis (C). For instance, $m=d_{1} \in \operatorname{cd}(G), m+1=d_{1} \in$ $\operatorname{cd}(H)$, and $m+2=d_{1} \in \operatorname{cd}(P)$, where $G, H$ and $P$ are solvable groups, then $m, m+1, m+2 \in \operatorname{cd}(G \times H \times P)$.

Remark 2 Even if $G$ is an insolvable group, we also cannot ascertain that $G$ is a product of $L$ by an abelian group. For example, if $d_{1}, d_{2}, d_{3} \in \operatorname{cd}(L)$ and $M$ is a nonabelian simple group such that $d_{1}(M)>d_{3}(L)$, then $d_{1}, d_{2}, d_{3} \in \operatorname{cd}(L \times M)$. Thus in Theorem 3 , the condition " $d_{i}(G)=d_{i}(L)$ for all $i \in\{1,2,3\}$, where $L$ is a nonabelian simple group", is essential and cannot be removed.

Remark 3 The condition " $G$ is almost simple" cannot be replaced by " $G$ is insolvable". For instance, for $n \geqslant 9, d_{1}\left(A_{n}\right)=n-1, d_{1}\left(A_{n+1}\right)=n$, and $d_{1}\left(A_{n+2}\right)=n+1$, then by Lemma $2, n-1, n, n+1 \in$ $\operatorname{cd}\left(A_{n} \times A_{n+1} \times A_{n+2}\right)$.

Corollary 1 Let $G$ be a nonabelian simple group with Hypothesis (C). Then $G$ is isomorphic to $L_{2}\left(2^{m}\right)$ with $m \geqslant 2$.

Proof: By Theorem $3, G / A \cong L_{2}\left(2^{m}\right)$ with $m \geqslant 2$. Since $G$ is simple, then $A=1$ and $G \cong L_{2}\left(2^{m}\right)$ with $m \geqslant 2$.

In this paper, we also use the properties of character degree graph $\Gamma(G)$ in the proofs of some results. So, we will introduce the notion of character degree graph $\Gamma(G)$. Let

$$
\rho(G)=\{p \in \pi(G) \mid p \text { divides } \chi(1), \chi \in \operatorname{Irr}(G)\} .
$$

Recall that the graph $\Gamma(G)$ is called character degree graph $^{13}$ whose vertices are members of $\rho(G)$ and two vertices $p$ and $q$ are joined by an edge if $p q$ divides some character degree of $G$, written as $p \sim q$.

\section{FINITE SIMPLE GROUP}

Lemma 1 Let $q-1, q, q+1 \in \operatorname{cd}(G)$ for a positive integer $q>1$. Then the order $|G|$ of $G$ is divisible by $\frac{1}{2} q\left(q^{2}-1\right)$.

Proof: Let $1<m \in \operatorname{cd}(G)$. Then by Theorem 3.11 of Ref. 4, there is a character $\chi \in \operatorname{Irr}(G)$ such that $m=\chi(1)$ and $m|| G \mid$. Since $(q-1, q+1)=2$ for odd $q$, and $(q-1, q+1)=1$ for even $q$, then $\frac{1}{2} q\left(q^{2}-1\right)$ divides the order $|G|$ of $G$.
Table 1 The first three smallest degrees of sporadic simple groups.

\begin{tabular}{lrrr}
\hline$G$ & $d_{1}(G)$ & $d_{2}(G)$ & $d_{3}(G)$ \\
\hline$M_{11}$ & 10 & 11 & 16 \\
$M_{12}$ & 11 & 16 & 45 \\
$J_{1}$ & 56 & 76 & 77 \\
$M_{22}$ & 21 & 45 & 55 \\
$J_{2}$ & 14 & 21 & 36 \\
$M_{23}$ & 22 & 45 & 230 \\
$H S$ & 22 & 77 & 154 \\
$J_{3}$ & 85 & 323 & 24 \\
$M_{24}$ & 23 & 45 & 231 \\
$M c L$ & 22 & 231 & 252 \\
$H e$ & 51 & 153 & 680 \\
$R u$ & 378 & 406 & 783 \\
$S u z$ & 143 & 364 & 780 \\
$O N$ & 10944 & 13376 & 25916 \\
$C o_{3}$ & 23 & 253 & 275 \\
$C o_{2}$ & 23 & 253 & 275 \\
$F i_{22}$ & 78 & 429 & 1001 \\
$H N$ & 133 & 760 & 3344 \\
$L y$ & 2480 & 45694 & 48174 \\
$M_{12}$ & 11 & 16 & 45 \\
$T h$ & 248 & 4123 & 27000 \\
$F i_{23}$ & 782 & 3588 & 5083 \\
$C o_{1}$ & 276 & 299 & 1771 \\
$J_{4}$ & 1333 & 299367 & 887778 \\
$F i_{24}^{\prime}$ & 8671 & 57477 & 249458 \\
$B$ & 4371 & 96255 & 1139374 \\
$M$ & 196883 & 21296876 & 842609326 \\
$2 F_{4}(2)^{\prime}$ & 26 & 27 & 78 \\
\hline & & &
\end{tabular}

Lemma 2 Let $G=A_{n}$ with $n \geqslant 9$. Then

(1) $d_{1}(G)=n-1$;

(2) $d_{2}(G)=\frac{1}{2} n(n-3)$;

(3) $d_{3}(G)=\frac{1}{2}(n-1)(n-2)$.

Proof: This results are taken from Ref. 14.

Lemma 3 Let $G$ be a sporadic simple group. Then $d_{i}(G)$ with $i \in\{1,2,3\}$ are as listed in Table 1 .

Proof: The results were obtained from ATLAS ${ }^{15}$.

Lemma 4 Let $G$ be a simple group of Lie type satisfying Hypothesis (C). Then $G$ is isomorphic to $L_{2}\left(2^{m}\right)$ with $m \geqslant 2$ or $L_{2}(5)$.

In the following proof of this Lemma, the results of Refs. 16, 17 will often be used without explicit reference.

Proof: Let $G$ be a simple group of Lie type. Then the following cases will be considered.

Case 1. Linear groups $L_{n}(q)$.

Let $n=2$ : 
(i) If $q=2^{m} \geqslant 4$, then $\operatorname{cd}(G)=\left\{1,2^{m}-1,2^{m}, 2^{m}+\right.$ $1\}$, and so $d_{1}(G)=2^{m}-1, d_{2}(G)=2^{m}$, and $d_{3}(G)=2^{m}+1$. Hence we have that $L_{2}\left(2^{m}\right)$ with $m \geqslant 2$, the desired result.

(ii) If $q=p^{m} \geqslant 5$ is odd, then $\operatorname{cd}(G)=\{1, q-$ $1, q, q+1,(q+\varepsilon) / 2\}$ with $\varepsilon=(-1)^{(q-1) / 2}$. If $q \equiv 1(\bmod 4)$, then $\operatorname{cd}(G)=\{1,(q+1) / 2, q-$ $1, q, q+1\}$, and so $d_{1}(G)=(q+1) / 2, d_{2}(G)=$ $q-1$, and $d_{3}(G)=q$. Thus $(q+1) / 2+1=$ $q-1$, and so $q=5$. If $q \equiv-1(\bmod 4)$, then $\operatorname{cd}(G)=\{1,(q-1) / 2, q-1, q, q+1\}$, and so $d_{1}(G)=(q-1) / 2, d_{2}(G)=q-1$, and $d_{3}(G)=q$. Thus $(q-1) / 2+1=q-1$ and $q=3 \ngtr 5$, a contradiction.

Let $n=3$ :

If $q=3$, then $\operatorname{cd}(G)=\{1,12,13,16,26,27,39\} ;$ if $q=4$, then $\operatorname{cd}(G)=\{1,20,35,45,63,64\}^{15}$. If $q \geqslant 5$, then by Ref. $18, \operatorname{cd}(G)=\left\{1, q^{3}, q(q+1)\right.$, $(q-1)^{2}\left(q^{2}+q+1\right),(q-1)\left(q^{2}+q+1\right), q^{2}+q+1$, $\left.(q+1)\left(q^{2}+q+1\right), \frac{1}{3}(q+1)\left(q^{2}+q+1\right)\right\}$, where the last degree appears only if $q \equiv 1(\bmod 3)$, and so $d_{1}(G)=q(q+1), d_{2}(G)=q^{2}+q+1$, and $d_{3}(G)=q^{3}-1$ when $q \not \equiv 1(\bmod 3)$, and $d_{1}(G)=q(q+1), d_{2}(G)=\frac{1}{3}(q+1)\left(q^{2}+q+1\right)$ and $d_{3}(G)=q^{3}-1$ when $q \equiv 1(\bmod 3)$. If $q \not \equiv 1(\bmod 3)$, then $q^{2}+q+2=q^{3}-1$ by the hypothesis, and so this equation has no solution in $\mathbb{N}$, since $q \geqslant 5$. If $q \equiv 1(\bmod 3)$, then $q(q+1)+1=\frac{1}{3}(q+1)\left(q^{2}+q+1\right)$, i.e., $q^{3}-q^{2}-q-2=0$, and so we obtain no answer in $\mathbb{N}$. So we rule out this case.

Let $n=4$ : Then $d_{1}(G)=q\left(q^{2}+q+1\right), d_{2}(G)=$ $(q+1)\left(q^{2}+1\right), d_{3}(G)=\left(q^{2}+q+1\right)(q-1)^{2}$. By the hypothesis, $d_{3}(G)-1=d_{2}(G)$, and so $\left(q^{2}+q+1\right)(q-$ $1)^{2}-1=(q+1)\left(q^{2}+1\right)$. It is easy to show that the equation has no solution in $\mathbb{N}$.

Let $n=5$ : Then $d_{1}(G)=q(q+1)\left(q^{2}+1\right), d_{2}(G)=$ $q^{4}+q^{3}+q^{2}+q+1, d_{3}(G)=q^{2}\left(q^{4}+q^{3}+q^{2}+q+1\right)$. Then the equation $q^{4}+q^{3}+q^{2}+q+1+1=q^{2}\left(q^{4}+\right.$ $q^{3}+q^{2}+q+1$ ) has no solution in $\mathbb{N}$.

Let $n \geqslant 6$ : Since $d_{1}(G)+1=d_{2}(G)$ by the hypothesis, we obtain that

$$
1=\frac{q^{n}-1}{q-1}\left(\frac{q^{n-1}-1}{q^{2}-1}-1\right) .
$$

Note that $n \geqslant 6$ and $q \geqslant 2,\left(q^{n}-1\right) /(q-1) \geqslant 1$ and $\left(q^{n-1}-1\right) /\left(q^{2}-1\right)-1 \geqslant 1$, so in this case, there is no solution in $\mathbb{N}$.

Case 2. Unitary groups $U_{n}\left(q^{2}\right)$ with $n \geqslant 3$ and $q \geqslant 3$.

If $n=3$, then by Ref. 18, $\operatorname{cd}(G)=\left\{1, q^{3},(q-\right.$ 1) $(q+1)^{2}, q(q-1), q^{2}-q+1,(q-1)\left(q^{2}-q+1\right), q\left(q^{2}-\right.$ $\left.q+1),(q+1)\left(q^{2}-q+1\right), \frac{1}{3}(q-1)\left(q^{2}-q+1\right)\right\}$, where the last degree appears only if $q \equiv-1(\bmod 3)$ and $d_{1}(G)=q(q-1), d_{2}(G)=\left(q^{2}-q+1\right), d_{3}(G)=(q-$ 1) $\left(q^{2}-q+1\right)$. Thus, by the hypothesis, $d_{2}(G)+1=$ $d_{3}(G)$, i.e., $(q-2)\left(q^{2}-q+1\right)=1$. It is easy to see that this equation has no solution in $\mathbb{N}$ since $q \geqslant 2$; so, this case is ruled out.

If $n=4$, then by Ref. 16, $d_{1}(G)=(q-1)\left(q^{2}+1\right)$, $d_{2}(G)=q\left(q^{2}-q+1\right), d_{3}(G)=\left(q^{2}+1\right)\left(q^{2}-q+1\right)$, and so $1=\left(q^{2}-q+1\right)^{2}$. Thus there is no solution in this equation in $\mathbb{N}$.

If $n=5$, then by Ref. 16, $d_{1}(G)=q(q-1)\left(q^{2}+\right.$ 1), $d_{2}(G)=q^{4}-q^{3}+q^{2}-q+1, d_{3}(G)=q^{2}\left(q^{4}-\right.$ $\left.q^{3}+q^{2}-q+1\right)$. It is easy to show that the equation $d_{2}(G)+1=d_{3}(G)$ has no solution in $\mathbb{N}$.

If $n=6$, then Ref. 16 implies that $d_{1}(G)=(q-$ 1) $\left(q^{2}+q+1\right)\left(q^{2}-q+1\right), d_{2}(G)=q\left(q^{4}-q^{3}+q^{2}-q+1\right)$, and $d_{3}(G)=\left(q^{2}-q+1\right)\left(q^{2}+q+1\right)\left(q^{4}-q^{3}+q^{2}-q+1\right)$. By the hypothesis, we have that

$$
1=\left(q^{4}-q^{3}+q^{2}-q+1\right)\left[\left(q^{2}-q+1\right)\left(q^{2}+q+1\right)-1\right],
$$

and the equation has no solution in $\mathbb{N}$.

If $n=7$, then we have that $d_{1}(G)=q(q-1)\left(q^{2}+\right.$ $q+1)\left(q^{2}-q+1\right), d_{2}(G)=q^{6}-q^{5}+q^{4}-q^{3}+q^{2}-q+1$, and $d_{3}(G)=q^{2}\left(q^{2}+1\right)\left(q^{6}-q^{5}+q^{4}-q^{3}+q^{2}-q+1\right)$. Now $d_{2}(G)+1=d_{3}(G)$ by the hypothesis, and we can see that that equation has no solution in $\mathbb{N}$.

Let $n \geqslant 8$. If $2 \nmid n$, then

$$
1=\left(\frac{q^{n}+1}{q+1}\right)\left(\frac{q^{n-1}-q^{2}}{q^{2}-1}-1\right) .
$$

It is easy to see that the equation has no solution in $\mathbb{N}$. If $2 \mid n$, then we have that $\frac{q^{n}+q}{q+1}+1=\frac{\left(q^{n}-1\right)\left(q^{n-1}+1\right)}{(q+1)\left(q^{2}-1\right)}$ when $q \neq 2$, and, by the hypothesis, that $\frac{q^{n}+q}{q+1}+1=$ $\frac{\left(q^{n}-1\right)\left(q^{n-1}-q\right)}{(q+1)\left(q^{2}-1\right)}$ when $q=2$. If $q \neq 2$, then the equation

$$
1=q\left(q^{2 n-2}-q^{n+1}+2 q^{n-1}-2 q^{2}+q-2\right)
$$

has no solution in $\mathbb{N}$ since $n \geqslant 8$. If $q=2$, then the left side of the equation

$$
\left(q^{n}+2 q+1\right)\left(q^{2}-1\right)=q\left(q^{n-2}\right)\left(q^{n}-1\right)
$$

is odd, where the right side of this equation is even. So we rule out this case.

Case 3. Orthogonal groups $B_{l}(q)$ with $q \geqslant 3$ and Symplectic groups $C_{l}(q)$.

Let $l=2$ :

(i) $q \equiv 0(\bmod 2)$. Then by Ref. $19, d_{1}(G)=q(q-$ $1)^{2} / 2, d_{2}(G)=q\left(q^{2}+1\right) / 2$, and $d_{3}(G)=q(q+$ $1)^{2} / 2$. By the hypothesis, $\frac{1}{2} q(q-1)^{2}+1=$ $\frac{1}{2} q\left(q^{2}+1\right)$ and so $q=1 \nsupseteq 3$, a contradiction. 
(ii) $q \equiv 1(\bmod 2)$. Then by Ref. $19, d_{1}(G)=q^{2}+1$, $d_{2}(G)=q(q-1)^{2} / 2$, and $d_{3}(G)=q(q+1)^{2} / 2$. By the hypothesis, $\frac{1}{2} q(q-1)^{2}+1=\frac{1}{2} q\left(q^{2}+1\right)^{2}$, and so there is no solution in $\mathbb{N}$.

Note that if $q$ is even and $l=2$, then $B_{2}(q) \cong C_{2}(q)$.

Let $l=3$. Subcase 1: $B_{3}(q)$.

If $q \equiv 0(\bmod 2)$, then $d_{1}(G)=q\left(q^{2}+q+1\right)(q-$ $1)^{2} / 2, d_{2}(G)=q\left(q^{2}+1\right)\left(q^{2}-q+1\right) / 2, d_{3}(G)=q\left(q^{2}-\right.$ $q+1)(q+1)^{2} / 2$. Since $d_{3}(G)-1=d_{2}(G)$, we have $1=\left(q^{2}-q+1\right) q^{2}$, and so the equation has no solution in $\mathbb{N}$.

If $q \equiv 1(\bmod 2)$, then $d_{1}(G)=\left(q^{2}+q+1\right)\left(q^{2}-\right.$ $q+1), d_{2}(G)=q\left(q^{2}+q+1\right)(q-1)^{2} / 2, d_{3}(G)=q\left(q^{2}-\right.$ $q+1)\left(q^{2}+1\right) / 2$. Since $d_{2}(G)-d_{1}(G)=1$, we obtain that $1=\left(q^{2}+q+1\right)\left[q(q-1)^{2} / 2-\left(q^{2}-q+1\right)\right]$ has no solution in $\mathbb{N}$ as $q \geqslant 3$.

Subcase 2: $C_{3}(q)$.

If $q \equiv 0,2(\bmod 4)$, then $d_{1}(G)=q\left(q^{2}+q+\right.$ 1) $(q-1)^{2} / 2, d_{2}(G)=q\left(q^{2}+1\right)\left(q^{2}-q+1\right) / 2, d_{3}(G)=$ $q\left(q^{2}-q+1\right)(q+1)^{2} / 2$. As $d_{3}(G)-d_{2}(G)=1$. By the hypothesis, we derive that $1=3 q^{2}\left(q^{2}-q+1\right) / 2$ has no solution in $\mathbb{N}$.

If $q \equiv 1(\bmod 4)$, then $d_{1}(G)=(q+1)\left(q^{2}-q+1\right)$, $d_{2}(G)=q\left(q^{2}+q+1\right)(q-1)^{2} / 2, d_{3}(G)=q\left(q^{2}+1\right)\left(q^{2}-\right.$ $q+1) / 2$. Since $d_{3}(G)-d_{2}(G)=1$, we have that $1=q^{3}$, a contradiction since $q$ is a prime-power.

If $q \equiv 3(\bmod 4)$, then $d_{1}(G)=(q-1)\left(q^{2}+q+1\right)$, $d_{2}(G)=q\left(q^{2}+q+1\right)(q-1)^{2} / 2, d_{3}(G)=q\left(q^{2}+1\right)\left(q^{2}-\right.$ $q+1) / 2$. Since $d_{2}(G)-d_{1}(G)=1$, we conclude that $1=\left(q^{3}-1\right)(q(q-1) / 2-1)$ has no solution in $\mathbb{N}$ since $q$ is a power of some prime.

Let $l=4$. Subcase 1: $B_{4}(q)$.

Now $d_{1}(G)=1 / 2 q\left(q^{2}+q+1\right)\left(q^{2}+1\right)(q-1)^{2}$, $d_{2}(G)=1 / 2 q\left(q^{2}-q+1\right)\left(q^{4}+1\right), d_{3}(G)=1 / 2 q\left(q^{2}+\right.$ $q+1)\left(q^{4}+1\right)$ for $q \equiv 0(\bmod 2)$ and $d_{1}(G)=\left(q^{2}+\right.$ 1) $\left(q^{4}+1\right), d_{2}(G)=1 / 2 q\left(q^{2}+q+1\right)\left(q^{2}+1\right)(q-1)^{2}$, $d_{3}(G)=1 / 2 q\left(q^{2}-q+1\right)\left(q^{4}+1\right)$ for $q \equiv 1(\bmod 2)$. By the hypothesis, $1=d_{3}(G)-d_{2}(G)$ implies that $1=q^{2}\left(q^{4}+1\right)$ and $1=q^{4}(2-q) / 2$. Clearly, these equations have no solutions in $\mathbb{N}$.

Subcase 2: $C_{4}(q)$.

$d_{1}(G)=1 / 2 q\left(q^{2}+q+1\right)\left(q^{2}+1\right)(q-1)^{2}, d_{2}(G)=$ $1 / 2 q\left(q^{2}-q+1\right)\left(q^{4}+1\right), d_{3}(G)=1 / 2 q\left(q^{2}+q+1\right)\left(q^{4}+\right.$ 1) for $q \equiv 1(\bmod 4)$ and $d_{1}(G)=q^{4}+1, d_{2}(G)=$ $1 / 2 q\left(q^{2}+q+1\right)\left(q^{2}+1\right)(q-1)^{2}, d_{3}(G)=1 / 2 q\left(q^{2}-\right.$ $q+1)\left(q^{4}+1\right)$ for $q \equiv 2,3(\bmod 4)$. Now as with the proof of $B_{4}(q)$, this case is ruled out.

Let $l=5$. Subcase 1: $B_{5}(q)$.

$d_{1}(G)=1 / 2 q\left(q^{2}+1\right)\left(q^{4}+q^{3}+q^{2}+q+1\right)(q-1)^{2}$, $d_{2}(G)=1 / 2 q\left(q^{4}-q^{3}+q^{2}-q+1\right)\left(q^{4}+1\right), d_{3}(G)=$ $1 / 2 q\left(q^{2}+1\right)\left(q^{4}-q^{3}+q^{2}-q+1\right)(q+1)^{2}$ for $q \equiv 0$ $(\bmod 2)$, and $d_{1}(G)=\left(q^{4}-q^{3}+q^{2}-q+1\right)\left(q^{4}+q^{3}+\right.$ $\left.q^{2}+q+1\right), d_{2}(G)=1 / 2 q\left(q^{2}+1\right)\left(q^{4}+q^{3}+q^{2}+q+\right.$
1) $(q-1)^{2}, d_{3}(G)=1 / 2 q\left(q^{4}-q^{3}+q^{2}-q+1\right)\left(q^{4}+1\right)$ for $q \equiv 1(\bmod 2)$. Now considering $d_{2}(G)-d_{1}(G)=1$, we can rule out this case.

Subcase 2: $C_{5}(q)$.

Now $d_{1}(G)=1 / 2 q\left(q^{2}+1\right)\left(q^{4}+q^{3}+q^{2}+q+1\right)(q-$ $1)^{2}, d_{2}(G)=1 / 2 q\left(q^{4}-q^{3}+q^{2}-q+1\right)\left(q^{4}+1\right), d_{3}(G)=$ $1 / 2 q\left(q^{2}+1\right)\left(q^{4}-q^{3}+q^{2}-q+1\right)(q+1)^{2}$ for $q \equiv$ $0,2(\bmod 4) ; d_{1}(G)=(q+1)\left(q^{4}-q^{3}+q^{2}-q+1\right)$, $d_{2}(G)=1 / 2 q\left(q^{2}+1\right)\left(q^{4}+q^{3}+q^{2}+q+1\right)(q-1)^{2}$, $d_{3}(G)=1 / 2 q\left(q^{4}-q^{3}+q^{2}-q+1\right)\left(q^{4}+1\right)$ for $q \equiv 1$ $(\bmod 4) ; d_{1}(G)=(q-1)\left(q^{4}+q^{3}+q^{2}+q+1\right), d_{2}(G)=$ $1 / 2 q\left(q^{2}+1\right)\left(q^{4}+q^{3}+q^{2}+q+1\right)(q-1)^{2}, d_{3}(G)=$ $1 / 2 q\left(q^{4}-q^{3}+q^{2}-q+1\right)\left(q^{4}+1\right)$ for $q \equiv 3(\bmod 4)$. Now considering the $d_{2}(G)-d_{1}(G)=1$, we rule out this case.

Similarly we illustrate that no group occurs when $l=6,7$.

The notation is taken from Ref. 17, and we will consider the case when the group is of a large rank. By Table 2, the following cases will be dealt with for $n \geqslant 6$.

Case 1: $S p_{2 n}(2)$ for $n>2$.

Now $d_{1}(G)=2^{n-1}\left(2^{n}-1\right), d_{2}(G)=2^{n-1}\left(2^{n}+1\right)$, and $d_{3}(G)=P_{1}$. By the hypothesis, $d_{2}(G)-d_{1}(G)=$ 1 , and so $1=2^{n}$, a contradiction since $n>2$.

Case 2: $S p_{2 n}(q)$ for $2 \mid q, q>2, n \geqslant 2$.

Now $d_{1}(G)=P_{1}, d_{2}(G)=q^{n}\left(q^{n}-1\right) / 2, d_{3}(G)=$ $q^{n}\left(q^{n}+1\right) / 2$. Now $d_{3}(G)-d_{2}(G)=1$ implies that $1=q^{n}$, and $n=0$, against $n \geqslant 2$.

Case 3: $P S p_{2 n}(q)$ for $2 \nmid q, n \geqslant 6$.

Now we have that $d_{1}(G)=\frac{q^{2 n}-1}{q-1}, d_{2}(G)=$ $\frac{\left(q^{n}-1\right)\left(q^{2 n-2}-1\right)}{(q-1)\left(q^{2}-1\right)}$ and $d_{3}(G)=N_{1}$. By the hypothesis, $d_{2}(G)-d_{1}(G)=1$ implies that

$$
1=\frac{q^{2 n}-1}{q-1}\left(\frac{q^{2 n-2}-1}{q^{2}-1}-1\right),
$$

and so this equation has no solution in $\mathbb{N}$.

Case 4: $\Omega_{2 n+1}(3)$ for $n \geqslant 4$.

Now $d_{1}(G)=q^{n}\left(q^{n}-1\right) / 2, d_{2}(G)=\frac{q^{2 n}-1}{q-1}$ and $d_{3}(G)=q^{n}\left(q^{n}+1\right) / 2$. By hypothesis, $d_{3}(G)-$ $d_{1}(G)=2$ implies that $2=q^{n}$, and so $q=2$ and $n=1$, against $n \geqslant 4$.

Case 5: $\Omega_{2 n+1}(q)$ for $2 \nmid q, q>3, n \geqslant 4$.

Now $d_{1}(G)=\frac{q^{2 n}-1}{q-1}, d_{2}(G)=q^{n}\left(q^{n}-1\right) / 2$, and $d_{3}(G)=q^{n}\left(q^{n}+1\right) / 2$. By the hypothesis, $d_{3}(G)-$ $d_{2}(G)=1$ shows that $1=q^{n}$, against $n \geqslant 4$.

Case 6: $P \Omega_{2 n}^{+}(2)$, for $n \geqslant 8$ or $P \Omega_{2 n}^{+}(3), n \geqslant 8$.

Now $d_{1}(G)=2^{n-1}\left(2^{n}-1\right), d_{2}(G)=\frac{\left(2^{n}-1\right)\left(2^{n-1}+1\right)}{q-1}$, $d_{3}(G)=2^{2 n-3}\left(2^{n}-1\right)\left(2^{n-1}-1\right) / 3 . d_{2}(G)-d_{1}(G)=1$ 
Table 2 The first three smallest degrees of $B_{l}$ or $C_{l}^{\dagger} !$

\begin{tabular}{lcccc}
\hline$G^{+}$ & Condition & $d_{1}(G)$ & $d_{2}(G)$ & $d_{3}(G)$ \\
\hline$S p_{4}(2)^{\prime}$ & & 6 & 10 & 15 \\
$S p_{4}(3)$ & $n>2$ & 27 & 36 & 40 \\
$S p_{2 n}(2)$ & $2 \mid q, q>2, n \geqslant 2$ & $2^{n-1}\left(2^{n}-1\right)$ & $2^{n-1}\left(2^{n}+1\right)$ & $\mathscr{P}_{1}$ \\
$S p_{2 n}(q)$ & $\mathscr{P}_{1}$ & $\frac{1}{2} q^{n}\left(q^{n}-1\right)$ & $\frac{1}{2} q^{n}\left(q^{n}+1\right)$ \\
$P S p_{4}(q)$ & $2 \nmid q, n=3,4,5$ & $\mathscr{P}_{1}$ & $\mathscr{P}_{n}$ & $\mathscr{P}_{2}$ \\
$P S p_{2 n}(q)$ & otherwise & $\mathscr{P}_{1}$ & $\mathscr{P}_{2}$ & $\mathscr{N}_{1}$ \\
\hline$\Omega_{2 n+1}(3)$ & $n \geqslant 4$ & $\mathscr{N}_{1}^{-}$ & $\mathscr{P}_{1}$ & $\mathscr{N}_{1}^{+}$ \\
$\Omega_{2 n+1}(q)$ & $2 \nmid q, q>3, n \geqslant 4$ & $\mathscr{P}_{1}$ & $\mathscr{N}_{1}^{+}$ & $\mathscr{N}_{2}^{-}$ \\
$P \Omega_{2 n}^{+}(2)$ & $n \geqslant 8$ & $\mathscr{N}_{1}$ & $\mathscr{P}_{1}$ & $\mathscr{P}_{2}$ \\
$P \Omega_{2 n}^{+}(3)$ & $n \geqslant 8$ & $\mathscr{N}_{1}$ & $\mathscr{N}_{1}$ & $\mathscr{P}_{2}$ \\
$P \Omega_{2 n}^{+}(q)$ & $\mathscr{P}_{1}$ & $\mathscr{N}_{2}$ \\
$P \Omega_{2 n}^{-}(q)$ & $n>3, n \geqslant 8$ & $\mathscr{P}_{1}$ & & $\mathscr{P}_{2}$ \\
\hline
\end{tabular}

$\dagger$ Obtained from Tables VII and VIII of Ref. 17.

$\star \mathscr{P}_{1}=\left(q^{2 n}-1\right) /(q-1), \mathscr{P}_{2}=\left(q^{2 n}-1\right)\left(q^{2 n-1}-1\right) /(q-1)\left(q^{2}-1\right), \mathscr{P}_{n}=(q+1)\left(q^{2}+1\right) \cdots\left(q^{n}+1\right)$ for $P S p_{2 n}(q)$ and $\Omega_{2 n+1}(q) ; \mathscr{P}_{1}=\left(q^{n}-\varepsilon\right)\left(q^{n-1}+\varepsilon\right) /(q-1), \mathscr{P}_{2}=\left(q^{n}-\varepsilon\right)\left(q^{2 n-2}-1\right)\left(q^{n-2}+\varepsilon\right) /(q-1)\left(q^{2}-1\right)$ for $P \Omega_{2 n}^{\varepsilon}(q) ; \mathscr{N}_{1}=$ $q^{2 n-2}\left(q^{2 n}-1\right)\left(q^{2}-1\right), \operatorname{dim} U=2$ for $P S p_{2 n}(q) ; \mathscr{N}_{1}^{+}=q^{n}\left(q^{n}+1\right) / 2, \mathscr{N}_{1}^{-}=q^{n}\left(q^{n}-1\right) / 2$ for $\Omega_{2 n+1}(q) ; \mathscr{N}_{1}=q^{n-1}\left(q^{n}-\right.$ $\varepsilon) / \operatorname{gcd}(2, q-1)$ for $P \Omega_{2 n}^{\varepsilon}(q) ; \mathscr{N}_{2}^{-}=2^{2 n-3}\left(2^{n}-1\right)\left(2^{n-1}-1\right) / 3$ for $\Omega_{2 n}^{+}(2)$.

implies that $1=\left(2^{n}-1\right)\left[\left(2^{n-1}+1\right) /(2-1)-2^{n-1}\right]$ and so $n=1$, a contradiction.

Similarly we can show that $P \Omega_{2 n}^{+}(3)$ does not satisfy Hypothesis (C).

Case 7: $P \Omega_{2 n}^{+}(q)$ for $q>3, n \geqslant 8$ or $P \Omega_{2 n}^{-}(q)$ for $n \geqslant 5$.

Then $d_{1}(G)=\frac{\left(q^{n}-1\right)\left(q^{n-1}+1\right)}{q-1}, d_{2}(G)=q^{n-1}\left(q^{n}-\right.$ $1) / \operatorname{gcd}(2, q-1), d_{3}(G)=\frac{\left(q^{n}-1\right)\left(q^{2 n-2}-1\right)\left(q^{n-1}+1\right)}{(q-1)\left(q^{2}-1\right)}$ for $\varepsilon=+$, and $d_{1}(G)=\frac{\left(q^{n}+1\right)\left(q^{n-1}-1\right)}{q-1}, d_{2}(G)=q^{n-1}\left(q^{n}+\right.$ $1) / \operatorname{gcd}(2, q-1), d_{3}(G)=\frac{\left(q^{n}+1\right)\left(q^{2 n-2}-1\right)\left(q^{n-1}-1\right)}{(q-1)\left(q^{2}-1\right)}$ for $\varepsilon=$ -. By the hypothesis, $d_{3}(G)-d_{1}(G)=2$ implies that

$$
2=\frac{\left(q^{n}-1\right)\left(q^{n-1}+1\right)}{q-1}\left(\frac{q^{2 n-2}-1}{q^{2}-1}-1\right)
$$

and

$$
2=\frac{\left(q^{n}+1\right)\left(q^{n-1}-1\right)}{q-1}\left(\frac{q^{2 n-2}-1}{q^{2}-1}-1\right) .
$$

It is easy to see that these equations have no solution in $\mathbb{N}$ since $n \geqslant 5$.

Case 4: exceptional simple groups of type ${ }^{2} E_{n}$, $E_{n}, F_{4}$, or ${ }^{2} F_{4}$.

For ${ }^{2} E_{6}(q), d_{1}(G)=q\left(q^{6}-q^{3}+1\right)\left(q^{4}+1\right)$, $d_{2}(G)=\left(q^{2}+q+1\right)\left(q^{6}-q^{3}+1\right)\left(q^{4}-q^{2}+1\right)\left(q^{2}-q+1\right)^{2}$, $d_{3}(G)=q^{2}\left(q^{2}+1\right)\left(q^{4}-q^{3}+q^{2}-q+1\right)\left(q^{4}+1\right)\left(q^{4}-\right.$ $\left.q^{2}+1\right)$, and $d_{2}(G)-d_{1}(G)=1$ implies that $1=$ $\left(q^{6}-q^{3}+1\right)\left[\left(q^{2}+q+1\right)\left(q^{4}-q^{2}+1\right)\left(q^{2}-q+1\right)^{2}-\right.$ $\left.q\left(q^{6}-q^{3}+1\right)\left(q^{4}+1\right)\right]$ has no solution in $\mathbb{N}$.

For $E_{6}(q), d_{1}(G)=q\left(q^{6}+q^{3}+1\right)\left(q^{4}+1\right), d_{2}(G)=$ $q^{2}\left(q^{2}+1\right)\left(q^{4}+q^{3}+q^{2}+q+1\right)\left(q^{4}+1\right)\left(q^{4}-q^{2}+1\right)$,
$d_{3}(G)=\left(q^{2}-q+1\right)\left(q^{6}+q^{3}+1\right)\left(q^{4}-q^{2}+1\right)\left(q^{2}+\right.$ $q+1)^{2}$ and since $d_{3}(G)-d_{2}(G)=1$, we obtain that $1=\left(q^{4}-q^{2}+1\right)\left[\left(q^{2}-q+1\right)\left(q^{6}+q^{3}+1\right)\left(q^{2}+q+\right.\right.$ $1)^{2}-q^{2}\left(q^{2}+1\right)\left(q^{4}+q^{3}+q^{2}+q+1\right)\left(q^{4}+1\right)$, so it has no solution in $\mathbb{N}$.

For $E_{7}(q), d_{1}(G)=q\left(q^{4}-q^{2}+1\right)\left(q^{6}+q^{5}+q^{4}+q^{3}+\right.$ $\left.q^{2}+q+1\right)\left(q^{6}-q^{5}+q^{4}-q^{3}+q^{2}-q+1\right), d_{2}(G)=q^{2}\left(q^{4}-\right.$ $\left.q^{2}+1\right)\left(q^{6}+q^{3}+1\right)\left(q^{6}-q^{3}+1\right)\left(q^{2}-q+1\right)^{2}\left(q^{2}+q+1\right)^{2}$, $d_{3}(G)=\left(q^{2}+q+1\right)\left(q^{4}+q^{3}+q^{2}+q+1\right)\left(q^{6}+q^{3}+\right.$ 1) $\left(q^{6}+q^{5}+q^{4}+q^{3}+q^{2}+q+1\right)\left(q^{6}-q^{5}+q^{4}-q^{3}+\right.$ $\left.q^{2}-q+1\right)(q-1)^{3}$. By hypothesis, $1=d_{3}(G)-d_{2}(G)$, and so it has no solution in $\mathbb{N}$ since the degree of the determinant $d_{3}(G)-d_{2}(G)$ is equal to 27 .

For $E_{8}(q), d_{1}(G)=q\left(q^{4}-q^{2}+1\right)\left(q^{8}-q^{6}+q^{4}-q^{2}+\right.$ 1) $\left(q^{4}+1\right)\left(q^{8}-q^{4}+1\right)\left(q^{2}+1\right)^{2}, d_{2}(G)=q^{2}\left(q^{8}-q^{6}+\right.$ $\left.q^{4}-q^{2}+1\right)\left(q^{4}+q^{3}+q^{2}+q+1\right)\left(q^{4}-q^{3}+q^{2}-q+1\right)\left(q^{6}+\right.$ $\left.q^{5}+q^{4}+q^{3}+q^{2}+q+1\right)\left(q^{6}-q^{5}+q^{4}-q^{3}+q^{2}-q+1\right)\left(q^{8}-\right.$ $\left.q^{7}+q^{5}-q^{4}+q^{3}-q+1\right)\left(q^{8}+q^{7}-q^{5}-q^{4}-q^{3}+q+1\right)$, $d_{3}(G)=\left(q^{4}-q^{2}+1\right)\left(q^{2}-q+1\right)\left(q^{2}+q+1\right)\left(q^{8}-q^{6}+\right.$ $\left.q^{4}-q^{2}+1\right)\left(q^{4}+q^{3}+q^{2}+q+1\right)\left(q^{4}-q^{3}+q^{2}-q+\right.$ 1) $\left(q^{4}+1\right)\left(q^{8}-q^{4}+1\right)\left(q^{8}-q^{7}+q^{5}-q^{4}+q^{3}-q+\right.$ 1) $\left(q^{8}+q^{7}-q^{5}-q^{4}-q^{3}+q+1\right)\left(q^{2}+1\right)^{2}$. By the hypothesis, $d_{3}(G)-d_{2}(G)=1$, and considering the degree of $d_{3}(G)-d_{2}(G)$, we rule out this case.

For $F_{4}(q), d_{1}(G)=\left(q^{2}+q+1\right)\left(q^{2}-q+1\right)\left(q^{4}-q^{2}+\right.$ $1), d_{2}(G)=1 / 2 q\left(q^{4}+1\right)(q-1)^{2}\left(q^{2}+q+1\right)^{2}, d_{3}(G)=$ $1 / 2 q\left(q^{2}+1\right)\left(q^{4}-q^{2}+1\right)\left(q^{4}+1\right)$ for $q \equiv 1,3,5,7,9,11$ $(\bmod 12)$, and $d_{1}(G)=1 / 2 q\left(q^{4}+1\right)(q-1)^{2}\left(q^{2}+\right.$ $q+1)^{2}, d_{2}(G)=1 / 2 q\left(q^{2}+1\right)\left(q^{4}+1\right)\left(q^{4}-q^{2}+1\right)$, $d_{3}(G)=1 / 2 q\left(q^{4}+1\right)(q+1)^{2}\left(q^{2}-q+1\right)^{2}$ for $q \equiv 2,4,8$ (mod 12). If the former, considering the degree of 
$d_{2}(G)-d-1(G)$, we rule out this case. If the latter, then $1=d_{2}(G)-d_{1}(G)=\left(q^{4}+1\right)\left[1 / 2 q\left(q^{2}+1\right)\left(q^{4}-\right.\right.$ $\left.\left.q^{2}+1\right)-1 / 2 q(q-1)^{2}\left(q^{2}+q+1\right)^{2}\right]$ has no solution in $\mathbb{N}$.

For ${ }^{2} F_{4}\left(q^{2}\right), d_{1}(G)=1 / 2 \sqrt{2}\left(-q^{2}+q^{4}+1\right)(q+$ 1) $(q-1) q\left(q^{2}+1\right)^{2}, d_{2}(G)=\left(-q^{2}+q^{4}+1\right)\left(q^{8}-q^{4}+\right.$ 1) $q^{2}, d_{3}(G)=(q-1)(q+1)\left(q^{8}-q^{4}+1\right)\left(q^{4}+1\right)^{2}$. Since $d_{3}(G)-d_{2}(G)$ is a determinant of degree 18 , the equation has no solution in $\mathbb{N}$.

In this case, there is no group satisfying Hypothesis (C).

Case 5: exceptional simple groups of type ${ }^{3} D_{4}(q)$.

Now $d_{1}(G)=q\left(q^{4}-q^{2}+1\right), d_{2}(G)=1 / 2 q^{3}\left(q^{4}\right.$ $\left.q^{2}+1\right)(q-1)^{2}, d_{3}(G)=1 / 2 q^{3}(q-1)^{2}\left(q^{2}+q+1\right)^{2}$ if $q \equiv 0(\bmod 2) ; d_{1}(G)=q\left(q^{4}-q^{2}+1\right), d_{2}(G)=$ $\left(q^{2}-q+1\right)\left(q^{2}+q+1\right)\left(q^{4}-q^{2}+1\right), d_{3}(G)=1 / 2 q^{3}\left(q^{4}-\right.$ $\left.q^{2}+1\right)(q-1)^{2}$ if $q \equiv 1(\bmod 2)$. It is easy to see that there does not exist a prime power $q$ such that $d_{1}(G)+1=d_{2}(G)$.

Case 6: exceptional simple groups of type ${ }^{2} G_{2}\left(q^{2}\right)$ or $G_{2}(q)$.

For ${ }^{2} G_{2}\left(q^{2}\right), d_{1}=\left(q^{2}+1-q \sqrt{3}\right)\left(q^{2}+1+q \sqrt{3}\right)$, $d_{2}=1 / 6 \sqrt{3} q(q-1)(q+1)\left(q^{2}+1-q \sqrt{3}\right), d_{3}=$ $1 / 6 \sqrt{3} q(q-1)(q+1)\left(q^{2}+1+q \sqrt{3}\right)$. Considering $d_{3}-d_{1}=2$, we can rule out this case.

For $G_{2}(q)$, we have five cases.

Case 1: $d_{1}=(q+1)\left(q^{2}-q+1\right), d_{2}=\left(q^{2}+q+\right.$ 1) $\left(q^{2}-q+1\right), d_{3}=1 / 6 q\left(q^{2}-q+1\right)(q-1)^{2}$ for $q \equiv 1$ $(\bmod 6)$.

Case 2: $d_{1}=(q-1)\left(q^{2}+q+1\right), d_{2}=1 / 6 q\left(q^{2}-\right.$ $q+1)(q-1)^{2}, d_{3}=1 / 6 q\left(q^{2}+q+1\right)(q+1)^{2}$ for $q \equiv 2$ $(\bmod 6)$.

Case 3: $d_{1}=\left(q^{2}+q+1\right)\left(q^{2}-q+1\right), d_{2}=$ $1 / 6 q\left(q^{2}-q+1\right)(q-1)^{2}, d_{3}=1 / 6 q\left(q^{2}+q+1\right)(q+1)^{2}$ for $q \equiv 3(\bmod 6)$.

Case 4: $d_{1}=(q+1)\left(q^{2}-q+1\right), d_{2}=1 / 6 q\left(q^{2}-\right.$ $q+1)(q-1)^{2}, d_{3}=1 / 6 q\left(q^{2}+q+1\right)(q+1)^{2}$ for $q \equiv 4$ $(\bmod 6)$.

Case 5: $d_{1}=(q-1)\left(q^{2}+q+1\right), d_{2}=\left(q^{2}+q+\right.$ 1) $\left(q^{2}-q+1\right), d_{3}=1 / 6 q\left(q^{2}-q+1\right)(q-1)^{2}$ for $q \equiv 5$ $(\bmod 6)$.

We will consider only case 1 because the other cases can be considered in a similar manner. By the hypothesis, $d_{2}(G)-d_{1}(G)=1$, and so $1=\left(q^{2}-q+\right.$ 1) $q(q-2)$. It is easy to see that it has no solution in $\mathbb{N}$ since $q$ is a prime-power. ${ }^{2} B_{2}(q)$.

Case 6: exceptional simple groups of type

We can obtain from Ref. 16 that $d_{1}(G)=$ $\sqrt{2} q\left(q^{2}-1\right) / 2, d_{2}(G)=\left(q^{2}-1\right)\left(q^{2}-\sqrt{2} q+1\right)$, and $d_{3}(G)=q^{4}$. By the hypothesis, $1=\left(q^{2}-1\right)\left[\left(q^{2}-\right.\right.$ $\sqrt{2} q+1)-\sqrt{2} q / 2]$, and it is easy to see that the equation has no solution in $\mathbb{N}$.

Lemma 5 An odd-order group $G$ does not satisfy Hypothesis (C).

Proof: Assume that the result is wrong. Then the $d_{i}(G)$ are odd. But by the hypothesis, $d_{2}(G)=1+$ $d_{1}(G)$ is even. By Theorem 3.11 of Ref. 4 , there is a character $\chi \in \operatorname{Irr}(G)$ such that $d_{2}(G)=\chi(1)$ divides $|G|$. It follows that $G$ is a group of an even order, a contradiction.

Theorem 4 Let $G$ be a nonabelian simple group with Hypothesis $(C)$. Then $G$ is isomorphic to $L_{2}\left(2^{m}\right)$ for $m \geqslant 2$.

Proof: By Classification Theorem of Finite Simple Groups, $G$ is isomorphic to an alternating group, a simple sporadic group or a simple group of Lie type.

Case 1: if $G$ is isomorphic to a simple group of Lie type, then $G$ is isomorphic to $L_{2}\left(2^{m}\right)$ with $m \geqslant 2$.

By Lemma $4, G$ is isomorphic to $L_{2}\left(2^{m}\right)$ with $m \geqslant 2$.

Case 2: if $G$ is isomorphic to a simple sporadic group, then there exists no group satisfying Hypothesis (C).

By Lemma 3, we can see that there does not exist a group satisfying Hypothesis (C).

Case 3: if $G$ is isomorphic to an alternating group, then $G \cong A_{5}$ is the desired result.

If $n \geqslant 9$, by Lemma 2, no group occurs.

If $n \leqslant 8$, then by Ref. 15 , we obtain $G \cong A_{5} \cong$ $L_{2}(5) \cong L_{2}(4)$, the needed result.

Remark 4 There are some groups of an odd order whose three odd smallest degrees are consecutive. For instance, $\operatorname{cd}(M)=\{1, n\}, \operatorname{cd}(N)=\{1, n+2\}$ and $\operatorname{cd}(P)=\{1, n+4\}$ where $n>1$ is an odd number, then $n, n+2, n+4 \in \operatorname{cd}(M \times N \times P)$.

\section{PROOF OF Theorem 3}

Lemma 6 Assume that $S_{i}$ are nonabelian simple groups and $N=S_{1} \times \cdots \times S_{l}$ is normal in $G$. Then for all $m \in \operatorname{cd}\left(S_{i}\right)$ and $n \in \operatorname{cd}\left(S_{j}\right)$ with $j \neq i, m n \in \operatorname{cd}(G)$.

Proof: If $m \in \operatorname{cd}\left(S_{i}\right)$ and $n \in \operatorname{cd}\left(S_{j}\right)$, then there are irreducible characters $\chi \in \operatorname{Irr}\left(S_{i}\right)$ and $\beta \in \operatorname{Irr}\left(S_{j}\right)$ such that $\chi(1)=m$ and $\beta(1)=n$. By Theorem 4.21 of Ref. 4, $\chi \beta \in \operatorname{Irr}(N)$ and so $(\chi \beta)(1)=m n \in$ $\operatorname{cd}(N) \subseteq \operatorname{cd}(G)$.

\section{Proof of Theorem 3}

Proof: By hypothesis, $d_{i}(G)=d_{i}(L)$ for all $i \in$ $\{1,2,3\}$. By Theorem $4, L \cong L_{2}\left(2^{m}\right)$ with $m \geqslant 2$ or 
$L_{2}(5)$. Let $q=2^{2 m}$ with $m \geqslant 2$. Then we have that

$$
|G|=\frac{1}{2}(q-1) q(q+1)
$$

and

$$
d_{1}(G)=q-1, \quad d_{2}(G)=q, \quad d_{3}(G)=q+1 .
$$

It follows that there are irreducible characters $\chi_{i}$ such that $\chi_{i}(1)=d_{i}$ for $i \in\{1,2,3\}$. Since $G$ is almost simple, then by Lemma 6 ,

$$
G / K \cong L_{2}(q)
$$

for some subgroup $K \unlhd G$. If $|G|=|L|$, then $K=1$ and $G \cong L$.

Theorem 5 There is no nonabelian simple group $G$ such that $d_{i}(G)$ are consecutive for all $i \in\{1,2, \ldots, n\}$ with $n \geqslant 4$.

Proof: Assume that there is a nonabelian simple group such that $d_{i}(G)$ are consecutive for all $i \in$ $\{1,2, \ldots, n\}$ with $n \geqslant 4$. Then the group satisfies Hypothesis (C). Now by Theorem 4, $G$ is isomorphic to $L_{2}\left(2^{m}\right)$ for $m \geqslant 2$. Since $\left|\operatorname{cd}\left(L_{2}\left(2^{m}\right)\right)\right|=4$ and $|c d(G)| \geqslant 5$, we obtain a contradiction.

\section{APPLICATIONS}

We can also obtain from the character degree graph some information about the character degrees of finite groups. As for the character degrees, Huppert ${ }^{20}$ illustrated the following conjecture.

Conjecture Let $G$ be a finite group, and let $H$ be a finite nonabelian simple group such that the set of character degrees of $G$ and $H$ are the same. Then $G \cong H \times A$, where $A$ is an abelian group.

Note that $L_{2}(4) \cong L_{2}(5)$. As an application of Theorem 3, we prove that the conjecture is true for the groups with Hypothesis (C).

Theorem 6 Let $G$ be a finite group and $H=L_{2}\left(2^{m}\right)$. If $\operatorname{cd}(G)=\operatorname{cd}(H)$, then $G \cong H \times A$ with $A$ an abelian group.

Proof: Since $\operatorname{cd}(G)=\left\{1,2^{m}-1,2^{m}, 2^{m}+1\right\}$ and $\left(2^{m}-1,2^{m}+1\right)=1$, we have that the degree graph $\Gamma(G)$ has three connected components, i.e., $s(G)=$ 3. If $G$ is solvable, then by Ref. $21, s(G) \leqslant 2$, a contradiction. Thus $G$ is insolvable. Clearly $G$ has Hypothesis (C). Then by Lemma 1 of Ref. 22, there is a normal series $1 \unlhd K \unlhd H \unlhd G$ such that

$$
H / K \cong \underbrace{L_{2}\left(2^{m}\right) \times \cdots \times L_{2}\left(2^{m}\right)}_{n}
$$

for a positive integer $n$. Assume that $n>1$. Recall that $d_{1}=2^{m}-1, d_{2}=2^{m}$, and $d_{3}=2^{m}+1$. Now Lemma 6 implies that for all $d_{i} \in \operatorname{cd}\left(L_{2}\left(2^{m}\right)\right), d_{i} d_{j} \in$ $\operatorname{cd}(G)$, a contradiction to the hypothesis. Thus we have $n=1$, hence $H / K$ is isomorphic to $L_{2}\left(2^{m}\right)$. It follows from that $L_{2}\left(2^{m}\right) \leqslant G / K \leqslant S L_{2}\left(2^{m}\right)$. Note that $L_{2}\left(2^{m}\right) \cong S L_{2}\left(2^{m}\right)$. Then $G / K \cong L_{2}\left(2^{m}\right)$ with $m \geqslant 2$.

Now let $\mathfrak{X}$ be the set of all direct product of mutually non-isomorphic nonabelian simple groups, and let $\mathfrak{D}$ be the set of all solvable groups. Clearly $\mathfrak{X}$ and $\mathfrak{D}$ satisfy Corollary 9.28 of Ref. 23. Now for each $G \in \mathfrak{X}$, $\operatorname{Aut}(G) / \operatorname{Inn}(G)$ is solvable. Then every extension of $L_{2}\left(2^{m}\right)$ by $K$ is isomorphic to $K \times L_{2}\left(2^{m}\right)$, i.e., $G \cong L_{2}\left(2^{m}\right) \times K$. If $K$ is nonabelian, then by Theorem 4.21 of Ref. $4, m d_{i} \in \operatorname{cd}(G)$ for $1 \neq$ $m \in \operatorname{cd}(K)$, a contradiction. Thus $K$ is abelian.

Note that Theorem 6 is also proved by Huppert $^{20}$. If we further consider group orders, then Theorem 6 implies the following result.

Theorem 7 Let $G$ be a finite group, and $H=L_{2}\left(2^{m}\right)$ with $m \geqslant 2$. Then $\operatorname{cd}(G)=\operatorname{cd}(H)$ and $|G|=|H|$ if and only if $G \cong H$.

Proof: $(\Longrightarrow)$ Since $\operatorname{cd}(G)=\left\{1,2^{m}-1,2^{m}, 2^{m}+1\right\}$, then $G$ has consecutive three smallest degrees of characters, and so, by Theorem $3, G / K$ is isomorphic to $L_{2}\left(2^{m}\right)$. Since $|G|=|H|$ and Lemma 6 , then we have that $K=1$. Hence $G$ is isomorphic to $L_{2}\left(2^{m}\right)$.

( $\Longrightarrow$ ) Since $G$ is isomorphic to $H$, then by Ref. $19, \operatorname{cd}(G)=\operatorname{cd}(H)$ and $|G|=|H|$, the desired result.

Acknowledgements: The project was supported by the Opening Project of Sichuan Province University Key Laborstory of Bridge Non-destruction Detecting and Engineering Computing (Grant Nos: 2019QYJ02 and 2018QYJ01), Postgraduate Research and Practice Innovation Programme of Jiangsu Province (Grant No: KYCX172012) and the second author was supported by NSF of China (Grant No: 11871360). The authors are very grateful for the helpful suggestions of the referees.

\section{REFERENCES}

1. Berkovich YG, Kazarin LS, Zhmud' EM (2018) Characters of Finite Groups, 2nd edn, Walter de Gruyter, Berlin.

2. Bianchi M, Chillag D, Mauri AG, Herzog M, Scoppola CM (1992) Applications of a graph related to conjugacy classes in finite groups. Arch Math Basel 58, 126-132.

3. Shao C, Jiang Q (2016) On normal subgroups with consecutive G-class sizes. J Algebra Appl 15, ID 1650151 . 
4. Isaacs IM (1994) Character Theory of Finite Groups, Dover Publications, New York.

5. Huppert B (1998) Character Theory of Finite Groups, Walter de Gruyter, Berlin.

6. Qian G, Shi W (2004) A note on character degrees of finite groups. J Group Theory 7, 187-196.

7. Aziziheris K (2013) Some character degree conditions implying solvability of finite groups. Algebr Represent Th 16, 747-754.

8. Jiang Q, Shao C (2014) Solvability of finite groups with four conjugacy class sizes of certain elements. Bull Aust Math Soc 90, 250-256.

9. Liu YJ, Liu Y (2016) Finite groups with exactly one composite character degree. J Algebra Appl 15, ID 1650132.

10. Shafiei F, Iranmanesh A (2016) The solvability comes from a given set of character degrees. J Algebra Appl 15, ID 1650164.

11. Aziziheris K, Yengejeh MHS (2017) Some solvable character degree sets. Comm Algebra 45, 4283-4291.

12. Qian G (2005) Finite groups with consecutive nonlinear character degrees. J Algebra 285, 372-382.

13. Manz O, Staszewski R, Willems W (1988) On the number of components of a graph related to character degrees. Proc Amer Math Soc 103, 31-37.

14. Rasala R (1977) On the minimal degrees of characters of $S_{n} . J$ Algebra 45, 132-181.
15. Conway JH, Curtis RT, Norton SP, Parker RA, Wilson RA (1985) Atlas of Finite Groups, Oxford University Press, UK.

16. Lübeck F (2007) Character degrees and their multiplicities for some groups of lie type of rank $<9$. Available at: www.math.rwth-aachen.de/ Frank. Luebeck/chev/DegMult/index.html?LANG=en.

17. Tiep PH, Zalesskii AE (1996) Minimal characters of the finite classical groups. Comm Algebra 24, 2093-2167.

18. Simpson WA, Frame JS (1973) The character tables for $\operatorname{SL}(3, q), \operatorname{SU}\left(3, q^{2}\right), \operatorname{PSL}(3, q), \operatorname{PSU}\left(3, q^{2}\right)$. Canad $J$ Math 25, 486-494.

19. Jordan HE (1907) Group-characters of various types of linear groups. American J Math 29, 387-405.

20. Huppert B (2000) Some simple groups which are determined by the set of their character degrees. Illinois $J$ Math 44, 828-842.

21. Manz O, Willems W, Wolf TR (1989) The diameter of the character degree graph. J Reine Angew Math 402, 181-198.

22. Xu H, Chen G, Yan Y (2014) A new characterization of simple $K_{3}$-groups by their orders and large degrees of their irreducible characters. Comm Algebra 42, 5374-5380.

23. Rose JS (1994) A Course on Group Theory, Dover Publications, New York. 